\title{
The influence of two-dimensional organic adlayer thickness on the ultralow frequency Raman spectra of transition metal dichalcogenide nanosheets
}

\author{
Shiyu $\mathrm{Wu}^{1}$, Xiaotong $\mathrm{Shi}^{1}$, Yue Liu ${ }^{1}$, Lin Wang ${ }^{1}$, Jindong Zhang ${ }^{1}$, Weihao Zhao ${ }^{1}$, Pei Wei ${ }^{1}$, \\ Wei Huang ${ }^{1,2}$, Xiao Huang ${ }^{1^{*}}$ and Hai $\mathrm{Li}^{1^{*}}$
}

\begin{abstract}
Recently, it has been reported that physisorbed adsorbates can be trapped between the bottom surface of twodimensional (2D) materials and supported substrate to form 2D confined films. However, the influence of such 2D confined adsorbates on the properties of $2 \mathrm{D}$ materials is rarely explored. Herein, we combined atomic force microscopy (AFM), Kelvin probe force microscopy (KPFM) and Raman spectroscopy especially the ultralow frequency (ULF) Raman spectroscopy to explore the influence of $2 \mathrm{D}$ confined organic adlayer thickness on the ULF breathing modes of few-layer $\mathrm{MoS}_{2}$ and $\mathrm{WSe}_{2}$ nanosheets. As the thickness of organic adlayers increased, red shift, coexistence of blue and red shifts as well as blue shift of ULF breathing mode was observed. KPFM measurement confirmed the enhanced $n$-doping and p-doping behaviors of organic adlayers as their thickness increased, respectively. Our results will provide new insights into the interaction between $2 \mathrm{D}$ confined adsorbates and bottom surface of $2 D$ nanosheets, which could be useful for modulating properties of $2 \mathrm{D}$ materials.
\end{abstract}

Keywords: two-dimensional organic adlayer, adlayer thickness, ultralow frequency Raman, transition metal dichalcogenides, atomic force microscopy

\section{INTRODUCTION}

Two-dimensional (2D) materials have quite large surface to volume ratio and thus their properties are inevitably affected by various physisorbed adsorbates on their top surface at ambient condition [1-19]. For instance, the adsorbed hydrogen atoms were demonstrated to open the zero bandgap of graphene [1]. Tunable bandgap engineering of graphene was also realized by physisorbed organic adsorbates [2]. In addition to graphene, physisorbed molecules also largely influenced the properties of $2 \mathrm{D}$ transition metal dichalcogenides (TMDCs). It has been reported that physisorbed p-type dopants enhanced while n-type dopants decreased the photoluminescence (PL) intensity of single-layer (1L) $\mathrm{MoS}_{2}$ [7,16]. The photoresponsivity and mobility of $\mathrm{MoS}_{2}$ nanosheet-based devices were highly influenced by physisorbed adsorbates on $\mathrm{MoS}_{2}$ nanosheets at ambient condition, such as $\mathrm{H}_{2} \mathrm{O}$ and $\mathrm{O}_{2}$ molecules $[8,20,21]$. Obvious photocurrent enhancement was also observed in $\mathrm{MoS}_{2}$ field-effect transistors (FETs) by depositing cesium carbonate on $\mathrm{MoS}_{2}$ surface [22]. Enhanced on/off current ratio of $\mathrm{MoS}_{2}$ nanosheet was obtained due to physisorption of benzyl viologen [10]. Both p-doping and $\mathrm{n}$-doping of $\mathrm{WSe}_{2}$ nanosheets were realized by physisorbed potassium and $\mathrm{NO}_{2}$, respectively $[4,5,12]$.

Aforementioned reports are based on the molecular physisorption on exposed top surface of $2 \mathrm{D}$ materials. Recently, it has been reported that $2 \mathrm{D}$ confined water and organic solvents film can be formed between the bottom surface of 2D materials and supported substrates [23-33]. Such confined 2D films also played important role in modulating properties of 2D materials. For example, ultrathin water film can be confined between graphene and mica substrate $[23,27]$. The charge transfer between graphene and mica substrate can be effectively screened by such confined water film [27]. Moreover, the sandwiched ultrathin water film can strongly decrease the PL of monolayer $\mathrm{MoS}_{2}$ by a factor of 30-50, which was attributed to trion formation $[29,34]$. In addition, the morphology of confined water played an important role in

${ }^{1}$ Key Laboratory of Flexible Electronics (KLOFE) \& Institute of Advanced Materials (IAM), Jiangsu National Synergetic Innovation Center for Advanced Materials (SICAM), Nanjing Tech University (NanjingTech), Nanjing 211816, China

${ }^{2}$ Shaanxi Institute of Flexible Electronics (SIFE), Northwestern Polytechnical University (NPU), Xi'an 710072, China

"Corresponding authors (emails: iamhli@njtech.edu.cn (Li H); iamxhuang@njtech.edu.cn (Huang X)) 
affecting the electronic structure of $\mathrm{MoS}_{2}$ [30]. Atomically thin water film was considered as n-type dopant while trapped water droplet acted as p-type dopant [29]. In recent years, $2 \mathrm{D}$ nanofluidics consisting of stacked 2D materials have been reported for energy conversion, water treatment and ion transport manipulation [35-40]. In the 2D nanofluidics, the charge density and membrane structure asymmetry could be influenced by confined adsorbates between $2 \mathrm{D}$ materials, resulting in enhanced ionic current rectification. Similarly, volatile small organic molecules can also be confined between $2 \mathrm{D}$ materials and substrate to form atomically thin films, which showed quite different properties from their bulk counterparts [24,28,31-33]. For instance, tetrahydrofuran (THF) molecules formed atomically thin films in a layerby-layer manner and exhibited both liquid and solid properties, which were confirmed by atomic force microscopy (AFM) [24]. Molecular dynamics simulation indicated that the first THF adlayer was viscous and exhibited liquid behavior while the second adlayer of THF showed solid-like behavior [28]. Due to the different interaction with mica, immobile crystal-like cyclohexane adlayers were confined between graphene and mica substrate [24,28]. Although great efforts have been involved to investigate the morphology evolution and dynamic structure behavior of confined atomically thin organic films, how the confined atomically thin organic adlayers influence the properties of $2 \mathrm{D}$ materials is remained unexplored.

In this work, volatile organic small molecules, such as acetone, ethanol and dichloromethane (DCM), were confined between mechanically-exfoliated $\mathrm{MoS}_{2}$ or $\mathrm{WSe}_{2}$ nanosheets and $\mathrm{SiO}_{2}$ substrates to form atomically thin adlayers. AFM measurement indicated that the thickness of organic adlayers was increased with increasing incubation time of $2 \mathrm{D}$ nanosheets exposed to organic vapors. Kelvin probe force microscopy (KPFM) and Raman spectroscopy especially the ultralow frequency (ULF) Raman spectroscopy were employed to explore the interaction between the organic adlayers and 2D nanosheets. KPFM measurement confirmed the p-type and n-type doping effect of organic adlayers on $\mathrm{MoS}_{2}$ nanosheets, respectively. Interestingly, we found that the thickness of organic adlayers, no matter n-type or p-type doping, showed obvious influence on the ULF breathing modes of few-layer $\mathrm{MoS}_{2}$ and $\mathrm{WSe}_{2}$ nanosheets with the same trend. For the adlayers with lower height $(<0.7 \mathrm{~nm}$ for acetone and $<0.4 \mathrm{~nm}$ for dichloromethane (DCM), respectively), red shift of ULF breathing modes was observed. Once the adlayers height was higher than a certain value $(1.6 \mathrm{~nm}$ for acetone and $0.8 \mathrm{~nm}$ for DCM), only blue shift of breathing mode peak was observed. If the adlayers height was in the range between them (from 0.7 to $1.6 \mathrm{~nm}$ for acetone and from $0.4 \mathrm{~nm}$ to $0.8 \mathrm{~nm}$ for DCM), coexistence of blue and red shift appeared. Moreover, we also found that the organic adlayers showed very good stability at ambient condition but can be greatly removed by annealing at high temperature. The interaction between confined organic adlayers and ULF breathing modes of few-layer TMDCs nanosheets may provide alternative method to modulate the properties of $2 \mathrm{D}$ materials.

\section{EXPERIMENTAL SECTION}

\section{Materials}

Acetone (99.8\%), dichloromethane (99.9\%) and ethanol (99.9\%) were obtained from J\&K Scientific Ltd. Singleand few-layer $\mathrm{MoS}_{2}$ and $\mathrm{WSe}_{2}$ nanosheets were isolated from $\mathrm{MoS}_{2}$ and $\mathrm{WSe}_{2}$ bulk crystals (HQ graphene, Netherlands). Silicon wafers were purchased from Bonda technology Pte. Ltd.

\section{Incubating TMDCs nanosheets in organic vapor environment}

Scheme 1 illustrates the whole experimental procedure. Firstly, $\mathrm{MoS}_{2}$ and $\mathrm{WSe}_{2}$ nanosheets were deposited onto freshly cleaned $90 \mathrm{~nm} \mathrm{SiO} / \mathrm{Si}$ substrates by mechanical exfoliation method (step a in Scheme 1). Then optical microscope (Axio Scope A1, Zeiss) was used to locate and image the few-layer TMDCs nanosheets. As shown in step b in Scheme 1, $\mathrm{MoS}_{2}$ and $\mathrm{WSe}_{2}$ nanosheets were characterized by ULF Raman spectroscope, AFM and KPFM. Organic solvent (acetone, ethanol or DCM) with volume of $\sim 15 \mathrm{~mL}$ was poured into a quartz dish. The quartz dish and TMDCs nanosheets on $\mathrm{SiO}_{2} / \mathrm{Si}$ substrate were put inside a quartz box with diameter of $70 \mathrm{~mm}$ and height of $40 \mathrm{~mm}$ (step c in Scheme 1). After that, the quartz box was sealed and stored at ambient condition $\left(22 \pm 2^{\circ} \mathrm{C}\right)$. After being incubated in organic vapor for some time, the TMDCs nanosheets were taken out of the sealed quartz container and characterized by ULF Raman spectroscope, AFM and KPFM (step $d$ in Scheme 1). In order to investigate the influence of organic adlayer with different thickness, some TMDCs nanosheets were incubated and taken out of the sealed quartz box for several cycles (step c, $d$ in Scheme 1). The organic adlayer trapped between the TMDCs nanosheet and substrate was shown in the magnified inset of step $\mathrm{d}$ in Scheme 1. 


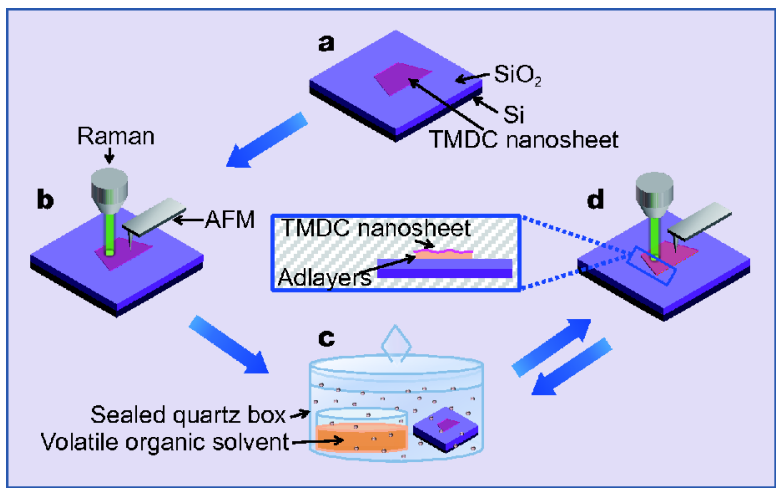

Scheme 1 The illustration of experimental procedures. (a) TMDCs nanosheet was deposited on $\mathrm{SiO}_{2}$ substrate by mechanical exfoliation. (b) AFM and Raman spectroscopy were used to characterize the morphology and ULF breathing mode of TMDCs nanosheet. (c) TMDCs nanosheet was incubated in a sealed quartz box filled with a small volume of volatile organic solvent for some time. (d) AFM and Raman spectroscopy were used to characterize the morphology and ULF breathing mode change of TMDCs nanosheet after being incubated in organic vapor environment. Magnified image in (d) clearly shows the structure of organic adlayer confined between TMDCs nanosheet and $\mathrm{SiO}_{2}$ substrate. Steps (c) and (d) might be repeated for several times to monitor the evolution and influence of organic adlayer.

\section{Raman characterization}

The Raman characterization was conducted in a backscattering configuration excited with a solid state green laser $(\lambda=532 \mathrm{~nm})$ (LabRAM HR Evolution, Horiba Jobin-Yvon). The Raman spectra of TMDCs nanosheets were collected through a $100 \times$ objective lens with accumulation time for $60 \mathrm{~s}$ and laser power of $1 \%$. The Raman band of a Si substrate at $520 \mathrm{~cm}^{-1}$ was used as a reference to calibrate.

\section{AFM and KPFM characterizations}

A commercial AFM instrument (Dimension ICON with Nanoscope V controller, Bruker) was used to image the samples in ScanAsyst mode in air. KPFM measurement was conducted in this AFM with a lift height of $80 \mathrm{~nm}$.

\section{RESULTS AND DISCUSSION}

The whole experimental process is illustrated in Scheme 1. After $\mathrm{MoS}_{2}$ or $\mathrm{WSe}_{2}$ nanosheets were deposited on $\mathrm{SiO}_{2}$ substrates by mechanical exfoliation (Scheme 1a), Raman spectrometer and AFM were used to monitor the morphology and Raman spectrum evolution of $\mathrm{MoS}_{2}$ or $\mathrm{WSe}_{2}$ nanosheets (Scheme $1 \mathrm{~b}$ ). As-prepared nanosheets were then put into a sealed quartz box filled with volatile organic solvents (acetone, ethanol or dichloromethane) and kept for a certain time (Scheme 1c). During this process, volatized organic molecules were permeated and trapped into the interface between TMDCs nanosheets and substrates to form atomically thin adlayers (magnified inset in Scheme 1d). After $\mathrm{MoS}_{2}$ or WSe $\mathrm{W}_{2}$ nanosheets were taken out of the sealed box, AFM and Raman spectrometer were used to check the morphology and Raman spectrum change (Scheme 1d). In order to investigate the influence of organic adlayer in detail, steps c and $\mathrm{d}$ were repeated in some experiments.

Monolayer (1L) $\mathrm{MoS}_{2}$ was firstly selected to investigate the influence of acetone adlayer on its morphology and Raman spectrum. As shown in Fig. 1a, $1 \mathrm{~L} \mathrm{MoS}_{2}$ had a height of $0.9 \mathrm{~nm}$ at ambient condition. KPFM measurement indicated that $1 \mathrm{~L} \mathrm{MoS}_{2}$ had a negative contact potential difference (CPD) of $-65 \mathrm{mV}$ compared with that of $\mathrm{SiO}_{2}$ substrate (Fig. 1g). The $\mathrm{MoS}_{2}$ nanosheet was then put into a sealed quartz box which contained acetone. Acetone has a saturated vapor pressure of $24.7 \mathrm{kPa}$ at $22^{\circ} \mathrm{C}$, and thus it is easy to generate acetone vapor at ambient condition. As shown in Fig. 1b, the height of $1 \mathrm{~L}$ $\mathrm{MoS}_{2}$ was increased to $1.5 \mathrm{~nm}$ after being exposed to acetone environment for $0.5 \mathrm{~h}$, indicating the thickness of acetone adlayer was around $0.6 \mathrm{~nm}$. Meanwhile, the CPD between $1 \mathrm{~L} \mathrm{MoS}_{2}$ and $\mathrm{SiO}_{2}$ substrate was increased to $-13 \mathrm{mV}$ as shown in Fig. $1 \mathrm{~h}$, indicating the $1 \mathrm{~L} \mathrm{MoS}_{2}$ was n-doped by acetone adlayer (referred to as AA- $\mathrm{MoS}_{2}$ ) $[16,29,41]$. The thickness of acetone adlayer was further increased to $0.8 \mathrm{~nm}$ after $1 \mathrm{~h}$ incubation (Fig. 1c), meaning more acetone molecules were trapped between $\mathrm{MoS}_{2}$ nanosheet and $\mathrm{SiO}_{2}$ substrate. In this case, there was almost no obvious potential difference between $\mathrm{MoS}_{2}$ and $\mathrm{SiO}_{2}$ substrate (Fig. 1i), indicating $1 \mathrm{~L} \mathrm{MoS}_{2}$ was further ntype doped by thicker acetone adlayer. As the incubation time was extended to $1.5,2$ and $2.5 \mathrm{~h}$, the thickness of acetone adlayer was increased to $1.1,1.3$ and $1.7 \mathrm{~nm}$ (Fig. $1 \mathrm{~d}-\mathrm{f}$ and $\mathrm{m}$ ), respectively. Meanwhile, the CPD between $\mathrm{MoS}_{2}$ and $\mathrm{SiO}_{2}$ substrate was simultaneously changed to 6, 19 and $28 \mathrm{mV}$ (Fig. $1 \mathrm{j}-\mathrm{l}$ ), respectively. Monotonic increase of the CPD between $1 \mathrm{~L} \mathrm{MoS}_{2}$ nanosheet and $\mathrm{SiO}_{2}$ substrate was observed from -65 to $28 \mathrm{mV}$ as the incubation time was increased from 0 to $2.5 \mathrm{~h}$ (Fig. $1 \mathrm{~m}$ ). It meant the work function of 1L AA-MoS2 nanosheet was increased about $0.093 \mathrm{eV}$ compared to that of pristine $1 \mathrm{~L}$ $\mathrm{MoS}_{2}$ nanosheet. The PL spectra of $1 \mathrm{~L} \mathrm{MoS}_{2}$ and AA$\mathrm{MoS}_{2}$ nanosheets are shown in Fig. 1n. The PL intensity of $1 \mathrm{~L} \mathrm{AA}-\mathrm{MoS}_{2}$ nanosheet was greatly decreased more than $90 \%$ even after being incubated in acetone environment for $0.5 \mathrm{~h}$ (Fig. 1o), which further confirmed the n-type doping of $\mathrm{MoS}_{2}$ nanosheet by acetone adlayer $[7,16]$. As the incubation time increased from 1 to $1.5 \mathrm{~h}$, the PL intensity of $1 \mathrm{~L} \mathrm{AA}-\mathrm{MoS}_{2}$ nanosheet was slightly 

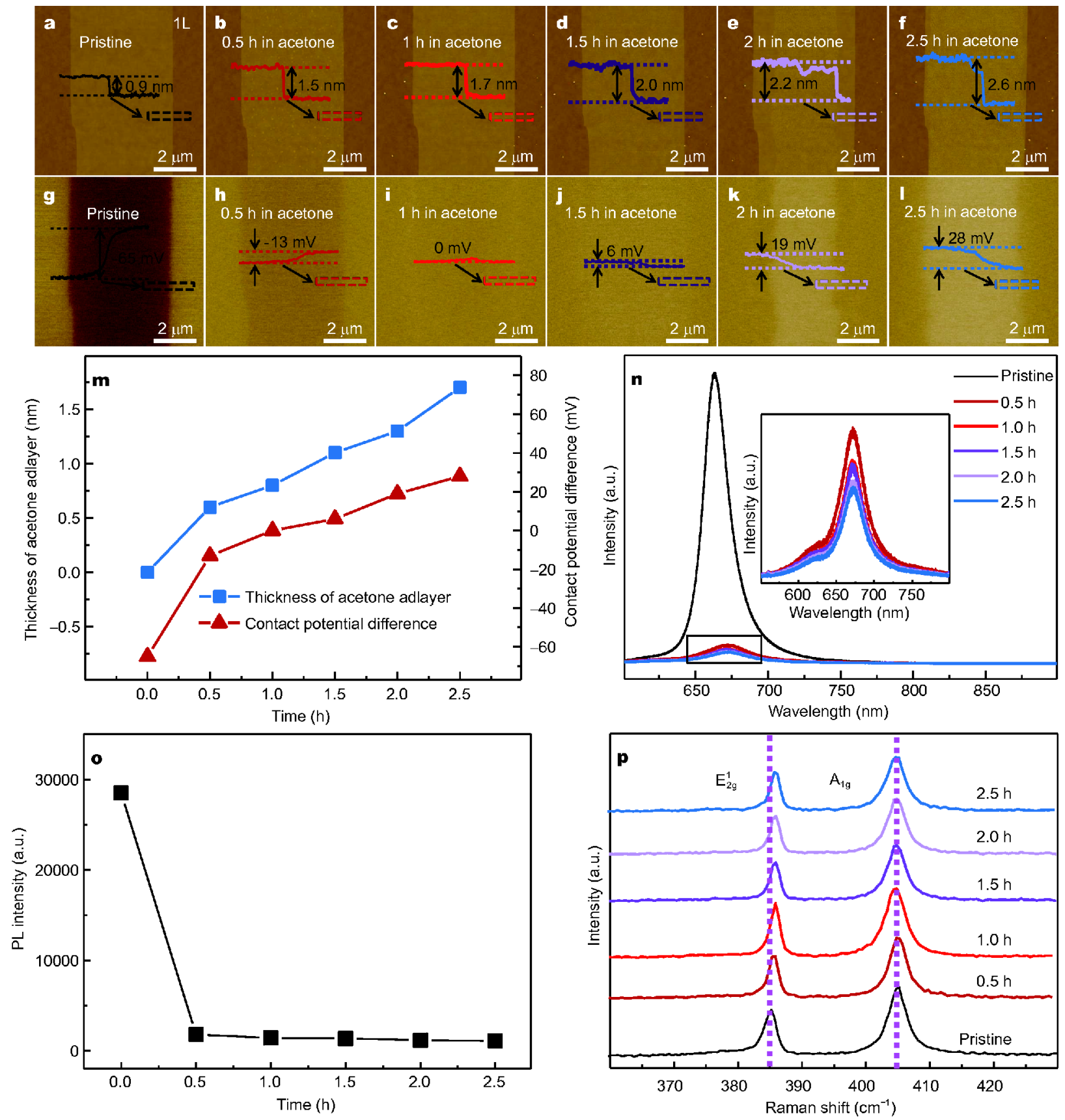

Figure 1 (a-f) AFM images of $1 \mathrm{~L} \mathrm{MoS}_{2}$ nanosheet (a) before and after being incubated in acetone environment for (b) 0.5, (c) 1, (d) 1.5, (e) 2 and (f) $2.5 \mathrm{~h}$, respectively. (g-l) Corresponding KPFM images of $1 \mathrm{~L} \mathrm{MoS}_{2}$ nanosheet (g) before and after being incubated in acetone environment for (h) 0.5 , (i) $1,(\mathrm{j}) 1.5$, (k) 2 and (l) $2.5 \mathrm{~h}$, respectively. (m) Plots of height and surface potential change of $1 \mathrm{~L} \mathrm{MoS}_{2}$ nanosheet with increased incubation time. (o) The PL intensity of $1 \mathrm{~L} \mathrm{MoS}_{2}$ nanosheet as a function of incubation time in acetone environment. (n, p) PL (n) and Raman (p) spectra of $1 \mathrm{~L}$ MoS 2 before and after being incubated in acetone environment.

decreased and kept almost unchanged after $2 \mathrm{~h}$. Raman spectroscopy was also used to characterize $1 \mathrm{~L} \mathrm{MoS}_{2}$ and AA- $\mathrm{MoS}_{2}$ nanosheets. As shown in Fig. 1p, the $\mathrm{E}_{2 \mathrm{~g}}^{1}$ peak of 1L AA- $-\mathrm{MoS}_{2}$ was blue-shifted by $0.8 \mathrm{~cm}^{-1}$ compared to that of pristine $1 \mathrm{~L} \mathrm{MoS}_{2}$, which is different from unchanged $\mathrm{E}_{2 \mathrm{~g}}^{1}$ peak of $1 \mathrm{~L} \mathrm{MoS}_{2}$ on water adlayer [29]. It has been reported that both electron and hole doping will not affect the position of $E_{2 \mathrm{~g}}^{1}$ peak [42], and thus this blue shift 
could be attributed to the stress induced by acetone adlayer $[8,43]$. There was no obvious change for $\mathrm{A}_{1 \mathrm{~g}}$ peak of 1L AA-MoS 2 , further implying the stress on $\mathrm{MoS}_{2}$ nanosheet induced by acetone adlayer [8].

In comparison with $E_{2 g}^{1}$ and $A_{1 g}$ peaks, ULF breathing Raman modes of $\mathrm{MoS}_{2}$ nanosheets are more sensitive to the surrounding environment [44]. As shown in Figs S1g and S2h-j in Supplementary information (SI), the ULF breathing modes of $2 \mathrm{~L}$ and $3 \mathrm{~L} \mathrm{MoS}_{2}$ were blue-shifted after being incubated in acetone environment, indicating the influence of acetone adlayer. However, the ULF breathing mode of $2 \mathrm{~L} \mathrm{MoS}_{2}$ is quite broad and that of $3 \mathrm{~L}$ $\mathrm{MoS}_{2}$ is overlapped by its strong shear mode. In order to clearly show the evolution of ULF breathing mode, thicker $\mathrm{MoS}_{2}$ nanosheets $(4 \mathrm{~L}-7 \mathrm{~L})$ were chosen to systematically investigate the influence of organic adlayer.

Fig. 2a, b show the AFM images of $6 \mathrm{~L} \mathrm{MoS}_{2}$ nanosheet before and after being incubated in acetone environment for $3 \mathrm{~h}$. The height of $\mathrm{MoS}_{2}$ nanosheet was increased from 4.4 to $4.9 \mathrm{~nm}$, indicating the thickness of confined acetone adlayer was $0.5 \mathrm{~nm}$. KPFM measurement showed the $\mathrm{CPD}$ between $6 \mathrm{~L} \mathrm{MoS}_{2}$ as well as $6 \mathrm{LMoS}_{2}$ /acetone adlayer (referred to as 6L AA- $-\mathrm{MoS}_{2}$ ) and $\mathrm{SiO}_{2}$ was increased from -94 to $-41 \mathrm{mV}$, confirming the $\mathrm{n}$-doping behavior of acetone adlayer on $6 \mathrm{~L} \mathrm{MoS}_{2}$ nanosheet [29]. It is consistent with that of $1 \mathrm{~L} \mathrm{MoS}_{2}$ nanosheet shown in Fig. 1g-l. Raman characterization showed that the $\mathrm{E}_{2 \mathrm{~g}}^{1}$ and $\mathrm{A}_{\mathrm{lg}}$ peaks of $6 \mathrm{~L} \mathrm{MoS}_{2}$ nanosheet kept unchanged before and after incubated in acetone environment (Figs S3-S5), indicating they are insensitive to acetone adlayer. However, a red shift of $0.6 \mathrm{~cm}^{-1}$ in ULF breathing mode was observed for $6 \mathrm{~L} \mathrm{AA}-\mathrm{MoS}_{2}$, implying there should be interaction between acetone adlayer and $6 \mathrm{LMoS}_{2}$. The red-shifted ULF breathing mode was named as B2 peak. Meanwhile, the PL spectrum of $6 \mathrm{~L} \mathrm{MoS}_{2}$ nanosheet was kept almost unchanged after being incubated in acetone environment (Fig. S6c).

After 6L $\mathrm{MoS}_{2}$ nanosheet was incubated in acetone environment for $4 \mathrm{~h}$, the thickness of $6 \mathrm{~L} \mathrm{MoS}_{2}$ nanosheet was increased from 4.3 to $5.5 \mathrm{~nm}$ (Fig. 3a, b), indicating the thickness of acetone adlayer was $1.2 \mathrm{~nm}$. In this case, the thick acetone adlayer may largely affect the properties of $\mathrm{MoS}_{2}$ nanosheet [45,46]. As shown in Fig. 3d, KPFM measurement indicated that the CPD of $6 \mathrm{~L} \mathrm{AA-MoS}$ was increased to $-6 \mathrm{mV}$ compared to that of pristine $6 \mathrm{~L} \mathrm{MoS}_{2}$, which was $-88 \mathrm{mV}$ (Fig. 3c). In comparison with the acetone adlayer with thickness of $0.5 \mathrm{~nm}$ (Fig. 2), this thicker acetone adlayer strongly affected the CPD of $\mathrm{MoS}_{2}$. The ULF breathing mode of 6L AA-MoS also showed obviously different Raman peak from that of

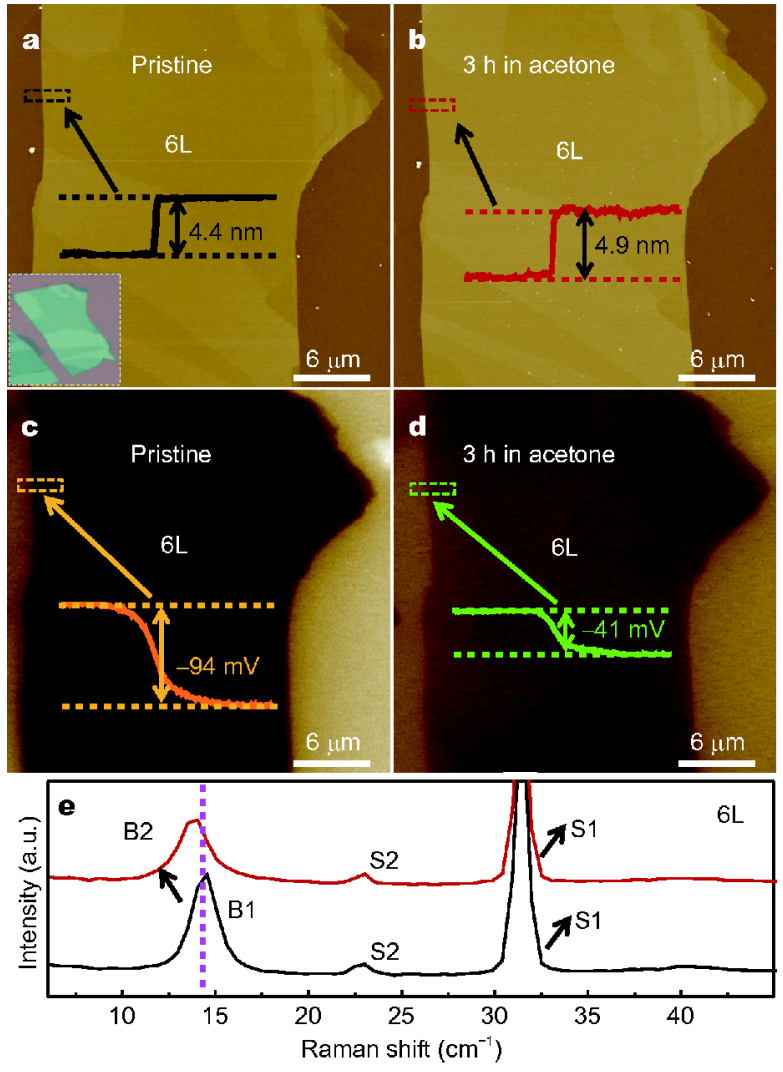

Figure 2 (a, b) AFM images of $6 \mathrm{~L} \mathrm{MoS}_{2}$ nanosheet (a) before and (b) after being incubated in acetone environment for $3 \mathrm{~h}$. Inset in (a) shows the optical image of $6 \mathrm{~L} \mathrm{MoS}$ nanosheet. (c, d) Corresponding KPFM images of $6 \mathrm{~L} \mathrm{MoS}_{2}$ nanosheet (c) before and (d) after being incubated in acetone environment. (e) ULF Raman spectra of $6 \mathrm{~L} \mathrm{MoS}_{2}$ before (black curve) and after (red curve) being incubated in acetone environment. Red shift of ULF breathing mode of $6 \mathrm{~L} \mathrm{MoS}_{2}$ nanosheet can be clearly observed.

pristine $6 \mathrm{~L} \mathrm{MoS}_{2}$, implying the significant role of acetone adlayer thickness. As shown in Fig. 3e, the ULF Raman

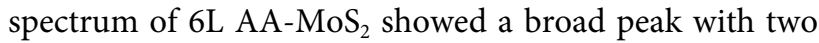
apexes around $14 \mathrm{~cm}^{-1}$, which disappeared under cross polarization (gray green curve in Fig. 3e), indicating it was breathing mode peak. The broad breathing mode peak can be deconvoluted into two Lorentzian profiles at $13.2 \mathrm{~cm}^{-1}$ (B2 peak) and $15 \mathrm{~cm}^{-1}$ (Fig. 3f), respectively. The peak located at $15 \mathrm{~cm}^{-1}$ blue-shifted compared to $\mathrm{B} 1$ peak and named as B3 peak. The B2 peak showed a red shift of $1 \mathrm{~cm}^{-1}$ while the B3 peak showed a blue shift of $0.8 \mathrm{~cm}^{-1}$ compared to that of pristine $6 \mathrm{~L} \mathrm{MoS}_{2}$ (B1 peak). The coexistence of red-shifted B2 peak and blue-shifted B3 peak might be attributed to the influence of thicker acetone adlayer. In order to exclude the influence of acetone adlayer, the top surface of another $6 \mathrm{~L} \mathrm{AA}-\mathrm{MoS}_{2}$ nanosheet was covered by a polydimethylsiloxane 

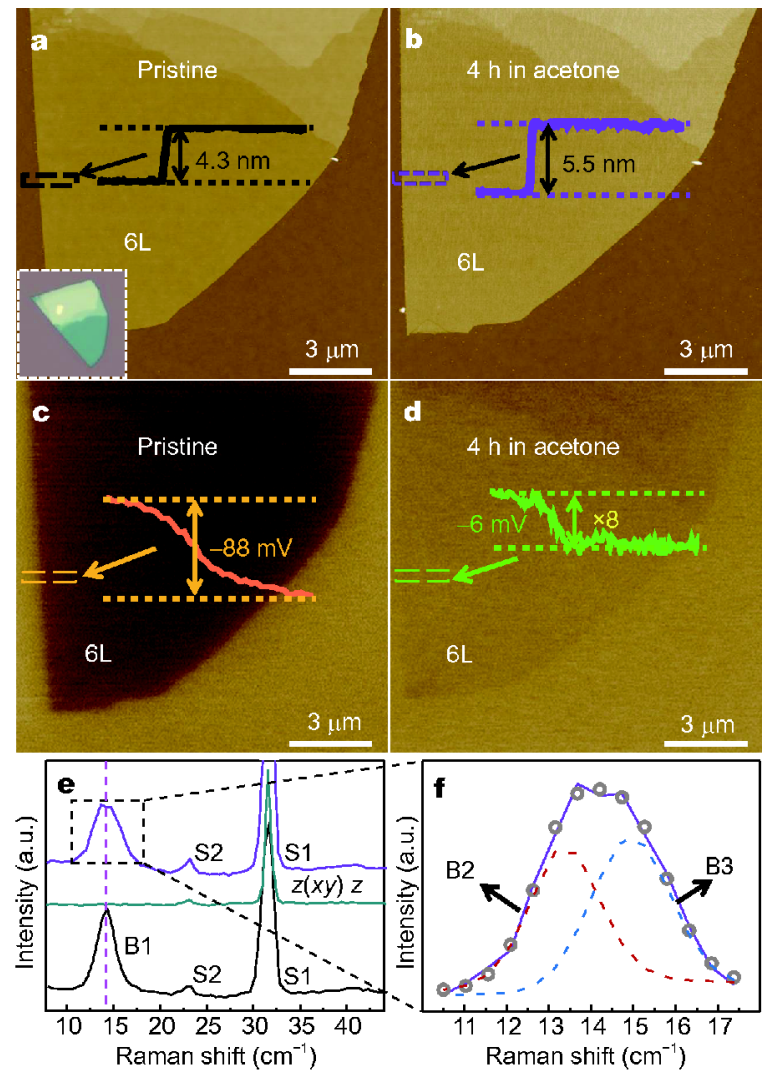

Figure 3 (a, b) AFM images of $6 \mathrm{~L} \mathrm{MoS}_{2}$ nanosheet (a) before and (b) after being incubated in acetone environment for $4 \mathrm{~h}$. Inset in (a) shows the optical image of $6 \mathrm{~L} \mathrm{MoS}_{2}$ nanosheet. (c, d) Corresponding KPFM images of $6 \mathrm{~L} \mathrm{MoS}_{2}$ nanosheet (c) before and (d) after being incubated in acetone environment. (e) ULF Raman spectra of $6 \mathrm{~L} \mathrm{MoS}_{2}$ before (black curve) and after (purple curve) being incubated in acetone environment. (f) Magnified image of ULF breathing mode of $\mathrm{MoS}_{2}$ nanosheet after being incubated in acetone environment. Coexistence of red and blue shift of ULF breathing mode of $6 \mathrm{~L} \mathrm{MoS}_{2}$ nanosheet can be clearly observed after deconvolution.

(PDMS) film and then peeled off from $\mathrm{SiO}_{2}$ substrate after being characterized by Raman spectroscopy. In this case, the bottom surface of $6 \mathrm{~L} \mathrm{MoS}_{2}$ nanosheet with trapped acetone adlayer was exposed to air and then acetone adlayer was evaporated quickly. Thus there was no trapped acetone adlayer between $\mathrm{MoS}_{2}$ nanosheet and PDMS film. As shown in Fig. S7, coexistence of red and blue shift of ULF breathing mode was observed for the $6 \mathrm{~L}$ AA- $\mathrm{MoS}_{2}$ nanosheet before transfer, which was similar to that of 6L AA-MoS2 nanosheet shown in Fig. 3e. However, the ULF breathing mode of $6 \mathrm{LMoS}_{2}$ recovered to its intrinsic position after being transferred on PDMS film, indicating the red and blue shift of ULF breathing mode was correlated to the existence of acetone adlayer. Besides 6L $\mathrm{MoS}_{2}$ nanosheet, the influence of acetone adlayer
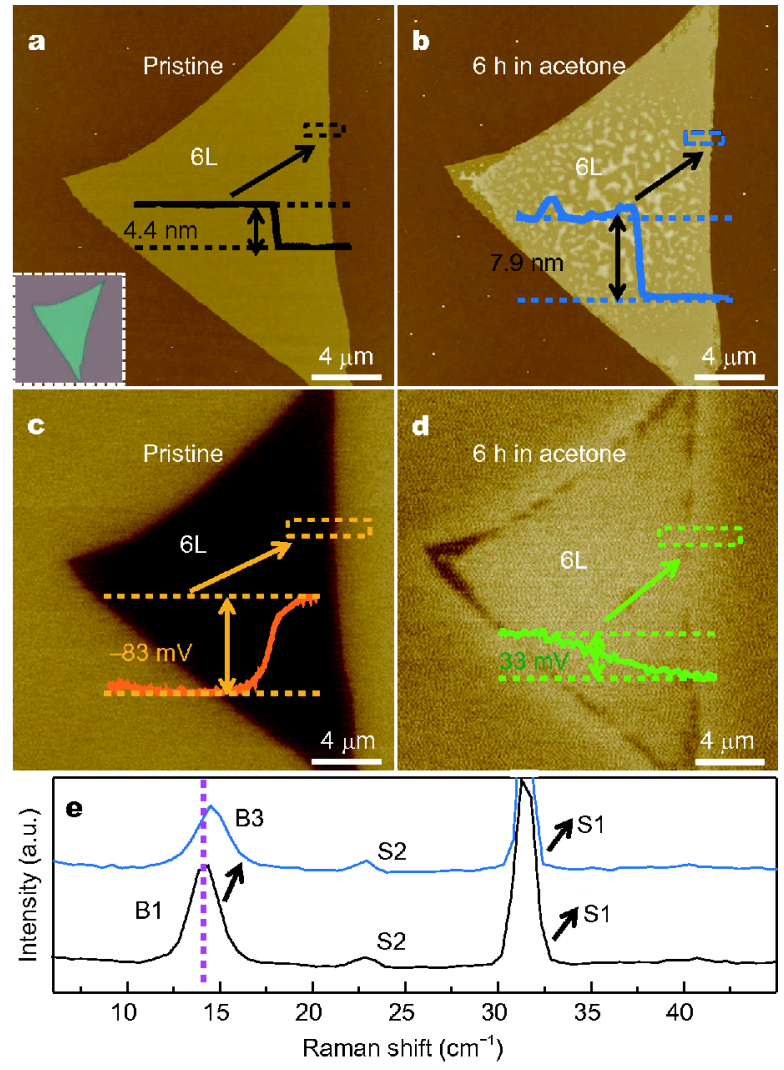

Figure 4 (a, b) AFM images of $6 \mathrm{~L} \mathrm{MoS}_{2}$ nanosheet (a) before and (b) after being incubated in acetone environment for $6 \mathrm{~h}$. Inset in (a) shows the optical image of $6 \mathrm{~L} \mathrm{MoS}_{2}$ nanosheet. (c, d) Corresponding KPFM images of $6 \mathrm{~L} \mathrm{MoS}_{2}$ nanosheet (c) before and (d) after being incubated in acetone environment. (e) ULF Raman spectra of $6 \mathrm{~L} \mathrm{MoS}_{2}$ before (black curve) and after (blue curve) being incubated in acetone environment. Blue shift of ULF breathing mode of $6 \mathrm{~L} \mathrm{MoS}_{2}$ nanosheet can be clearly observed.

thickness on the ULF breathing mode was also observed in $4 \mathrm{~L}, 5 \mathrm{~L}$ and $7 \mathrm{~L} \mathrm{MoS}_{2}$ nanosheets (Figs S8-S14), implying the versatile role of acetone adlayer thickness on the ULF breathing mode.

The thickness of acetone adlayer can be further increased by extending the incubation time. As shown in Fig. $4 \mathrm{a}, \mathrm{b}$, the thickness of acetone adlayer was increased to $3.5 \mathrm{~nm}$ after being incubated in acetone environment for $6 \mathrm{~h}$. With increased thickness, the influence of acetone adlayer on the properties of $\mathrm{MoS}_{2}$ was also enhanced. As shown in Fig. 4c, d, the CPD of 6L AA-MoS 2 was changed to positive value and showed an increment of $116 \mathrm{mV}$ compared to that of pristine $6 \mathrm{~L} \mathrm{MoS}_{2}$ nanosheet. The Raman spectrum of $6 \mathrm{~L}$ AA- $-\mathrm{MoS}_{2}$ showed ULF breathing mode peak at $14.6 \mathrm{~cm}^{-1}$ (B3 peak), which showed a blue shift of $0.4 \mathrm{~cm}^{-1}$ compared to that of pristine $6 \mathrm{~L} \mathrm{MoS}_{2}$ nanosheet (Fig. 4e). Such blue shift could be attributed to 

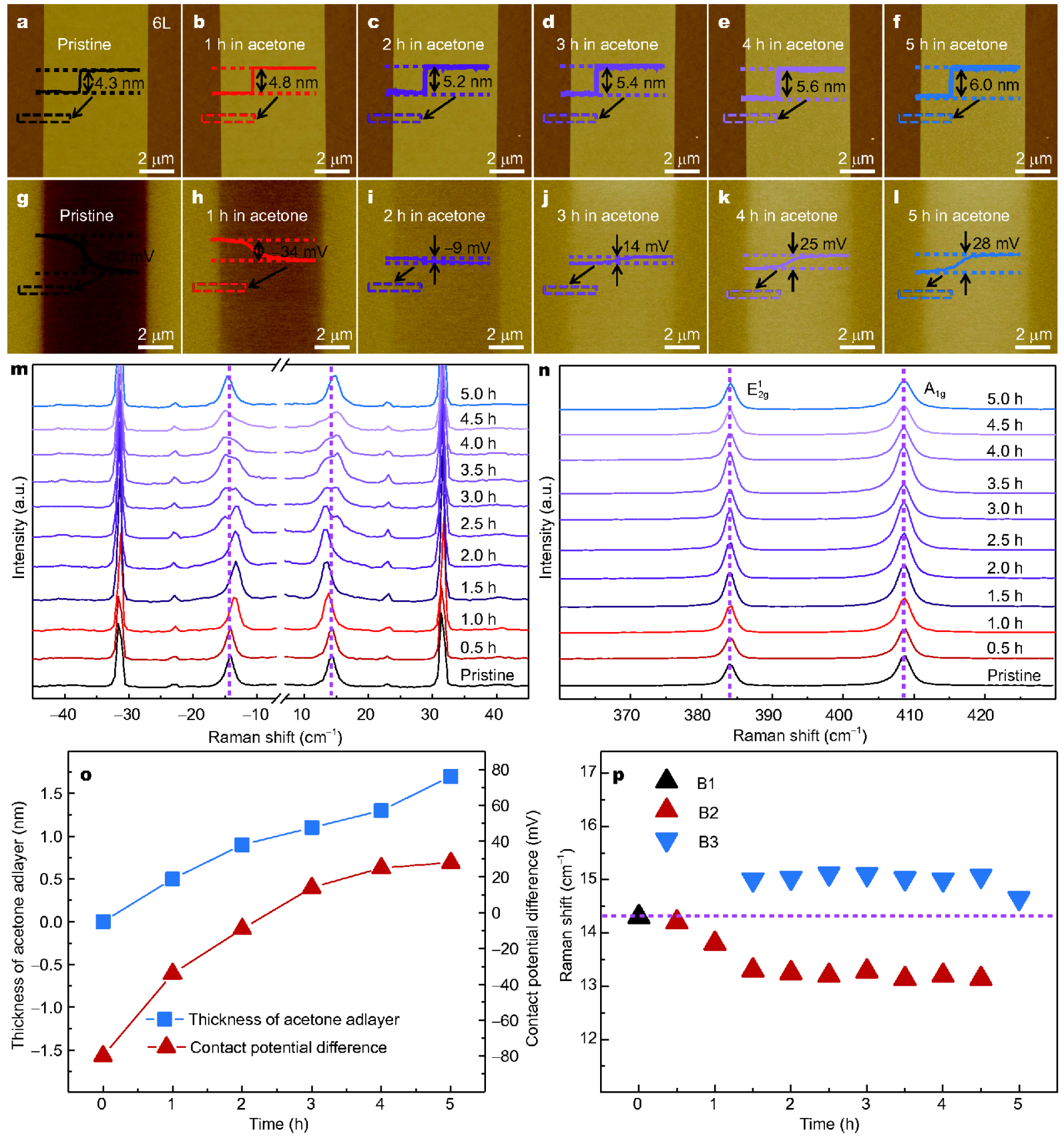

Figure 5 (a-f) AFM images of $6 \mathrm{~L} \mathrm{MoS}_{2}$ nanosheet (a) before and after being incubated in acetone environment for (b) 1, (c) 2, (d) 3, (e) 4 and (f) $5 \mathrm{~h}$, respectively. (g-1) Corresponding KPFM images of $1 \mathrm{~L} \mathrm{MoS}_{2}$ nanosheet (g) before and after being incubated in acetone environment for (h) 1 , (i) 2, (j) 3, (k) 4 and (l) 5 h, respectively. (m, n) ULF (m) and high frequency (n) Raman spectra of $6 \mathrm{~L} \mathrm{MoS}{ }_{2}$ before and being incubated in acetone environment. (o) Plots of height and surface potential change of $6 \mathrm{~L} \mathrm{MoS}_{2}$ nanosheet with increased incubation time. (p) Evolution of B2 and B3 peaks with increased incubation time.

the strong influence of thick acetone adlayer.

In order to monitor the evolution of acetone adlayer at different incubation time, AFM and Raman characterizations were performed on the same $\mathrm{MoS}_{2}$ nanosheet. Fig. $5 \mathrm{a}-\mathrm{f}$ show the AFM images of $6 \mathrm{~L} \mathrm{MoS}_{2}$ nanosheet before and after being incubated in acetone environment for $1,2,3,4$ and $5 \mathrm{~h}$, respectively. The height of pristine $6 \mathrm{~L} \mathrm{MoS}_{2}$ nanosheet was measured as $4.3 \mathrm{~nm}$. While the thickness of acetone adlayer was increased from 0.5, 0.9, $1.1,1.3$ to $1.7 \mathrm{~nm}$ as the incubation time is $1,2,3,4$ and 


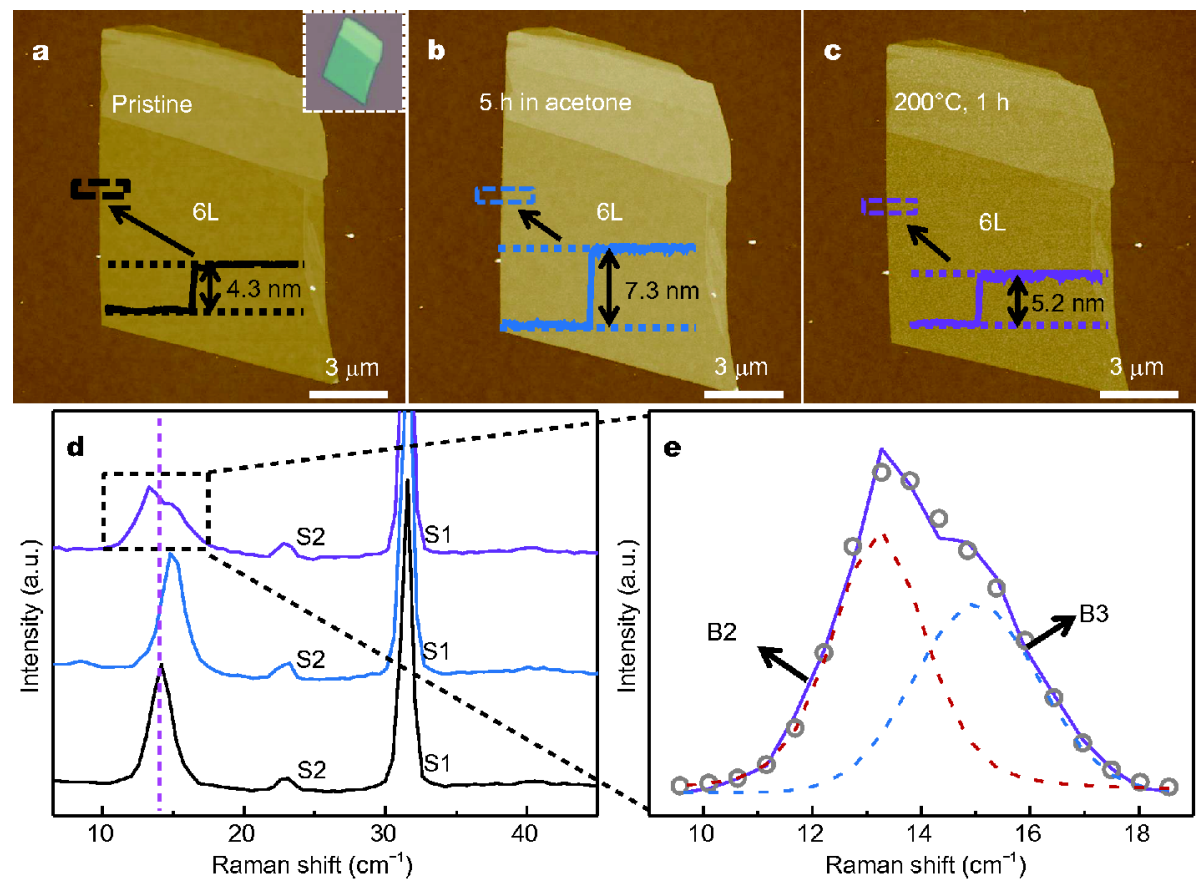

Figure 6 (a, b) AFM images of $6 \mathrm{~L} \mathrm{MoS}_{2}$ nanosheet (a) before and (b) after being incubated in acetone environment. (c) AFM image of 6L MoS 2 nanosheet shown in (b) after being annealed at $200^{\circ} \mathrm{C}$ for $1 \mathrm{~h}$. (d) ULF Raman spectra of $6 \mathrm{~L} \mathrm{MoS}_{2}$ before (black curve) and after being incubated in acetone (blue curve) environment as well as annealed (purple curve). (e) Magnified image of ULF breathing mode of MoS ${ }_{2}$ nanosheet shown in (d). Coexistence of red and blue shift of ULF breathing mode of $6 \mathrm{~L} \mathrm{MoS}_{2}$ nanosheet can be clearly observed after deconvolution.

$5 \mathrm{~h}$ (Fig. 5o), respectively. As shown in Fig. 5m, the ULF Raman spectra of pristine $6 \mathrm{~L} \mathrm{MoS}_{2}$ and $\mathrm{AA}-\mathrm{MoS}_{2}$ at different incubation time clearly showed the change of breathing mode. Meanwhile, the high frequency Raman spectra of pristine $6 \mathrm{~L} \mathrm{MoS}_{2}$ and AA-MoS 2 remained unchanged (Fig. $5 \mathrm{n}$ ). After being incubated in acetone environment for $0.5 \mathrm{~h}$, the $\mathrm{B} 1$ peak showed no notable change. While the ULF breathing mode peak of AA- $\mathrm{MoS}_{2}$ (B2 peak) showed a red shift of $0.5 \mathrm{~cm}^{-1}$ compared to that of pristine $\mathrm{MoS}_{2}$ (B1 peak) after $1 \mathrm{~h}$ incubation. The B2 peak kept red-shifting as the incubation time was increased to $1.5 \mathrm{~h}$. Meanwhile, a weak B3 peak was observed (Fig. S15). An obvious B3 peak appeared when the incubation time was increased to $2.5 \mathrm{~h}$ (Fig. S15c). The intensity ratio of $\mathrm{B} 2$ to $\mathrm{B} 3$ further decreased with the incubation time increased to $4.5 \mathrm{~h}$. After $5 \mathrm{~h}$ incubation, B2 peak disappeared and only B3 peak can be observed. Fig. $5 p$ clearly shows the evolution of B1, B2 and B3 peaks with varied incubation time. KPFM measurement was also performed simultaneously to show the CPD change of $\mathrm{MoS}_{2}$ nanosheet (Fig. 5g-l). As shown in Fig. 5o, the CPD kept increasing with increased incubation time, i.e., increased thickness of acetone adlayer, which is consistent with aforementioned results.
As-trapped acetone adlayer is quite stable at ambient condition. As shown in Fig. S16a, b, the height of pristine 6L $\mathrm{MoS}_{2}$ was measured as $4.2 \mathrm{~nm}$ and then increased to $5.3 \mathrm{~nm}$ after being incubated in acetone environment for $3 \mathrm{~h}$. The 6L AA-MoS ${ }_{2}$ nanosheet was then taken out and stored at ambient condition. There was no height change even after 169 h (Fig. S16c-g). ULF Raman spectroscopy was also used to monitor this process. As shown in Fig. S16h, there was a red-shifted breathing mode peak with a

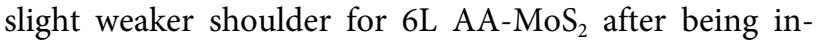
cubated in acetone environment for $3 \mathrm{~h}$. After deconvolution, the red-shifted breathing mode peak and the shoulder peak can be assigned as B2 and B3, respectively. These two peaks were kept unchanged at ambient condition even after $169 \mathrm{~h}$, indicating the stability of acetone adlayer.

In order to explore the reverse influence of acetone adlayer thickness on ULF breathing mode, a $6 \mathrm{~L} \mathrm{MoS}_{2}$ nanosheet was firstly incubated in acetone environment and then annealed at $200^{\circ} \mathrm{C}$. As shown in Fig. $6 \mathrm{a}, \mathrm{b}$, the height of $\mathrm{MoS}_{2}$ nanosheet was increased from 4.3 to $7.3 \mathrm{~nm}$ after being incubated in acetone environment for $5 \mathrm{~h}$, indicating the thickness of acetone adlayer was $3.0 \mathrm{~nm}$. Similar to the data shown in Fig. 4, the 6L AA- 
$\mathrm{MoS}_{2}$ showed a blue-shifted ULF breathing mode peak due to the thick acetone adlayer (Fig. 6d), which is consistent with the data shown in Fig. 4. As shown in Fig. 6c, the thickness of acetone adlayer was reduced to $0.9 \mathrm{~nm}$ after the $6 \mathrm{~L} \mathrm{AA}-\mathrm{MoS}_{2}$ nanosheet was annealed at $200^{\circ} \mathrm{C}$ for $1 \mathrm{~h}$, indicating the trapped acetone adlayer can be partially removed under high temperature. Meanwhile, the ULF breathing mode of $\mathrm{MoS}_{2}$ showed a broad peak located around $14 \mathrm{~cm}^{-1}$, which can also be deconvoluted into two Lorentzian profiles (Fig. 6e). In addition, 6L $\mathrm{MoS}_{2}$ with acetone adlayer of middle thickness was also annealed to confirm the reversibility. As shown in Fig. $\mathrm{S} 17$, the thickness of acetone adlayer was $1.2 \mathrm{~nm}$ after being incubated in acetone environment for $3 \mathrm{~h}$. In this case, the 6L AA- $\mathrm{MoS}_{2}$ showed a broad ULF breathing mode peak. After being annealed at $200^{\circ} \mathrm{C}$ for $1 \mathrm{~h}$, the thickness of acetone adlayer was decreased from 1.2 to $0.3 \mathrm{~nm}$. Meanwhile, the ULF breathing mode of $\mathrm{MoS}_{2}$ showed a red shift of $0.4 \mathrm{~cm}^{-1}$, which is consistent with the data shown in Fig. 2. Similar results were also observed in $7 \mathrm{~L} \mathrm{MoS}_{2}$ nanosheet annealed at different temperature (Fig. S18). Finally, the acetone adlayer can be completely removed by annealing at $300^{\circ} \mathrm{C}$ for $3 \mathrm{~h}$ (Fig. S18). Aforementioned results proved that the thickness of organic adlayer played an important role in affecting the ULF breathing mode of $\mathrm{MoS}_{2}$ nanosheet.

Besides acetone, ethanol adlayer also showed n-type doping behavior and similar influence on the ULF breathing mode of $\mathrm{MoS}_{2}$ nanosheet (Figs S19, S20) [7]. The PL spectrum of $1 \mathrm{~L} \mathrm{MoS}_{2}$ nanosheet was largely decreased after being incubated in ethanol environment for 1 and $2 \mathrm{~h}$ (Fig. S19a), respectively, confirming the n-type doping behavior of ethanol. The ULF breathing mode of $4 \mathrm{~L}$ and $6 \mathrm{~L} \mathrm{MoS}_{2}$ nanosheets showed red shift, coexistence of red and blue shift, and blue shift with increased incubation time in ethanol environment (Fig. S20), respectively. In contrast to acetone and ethanol, chloromethane was considered as p-type dopant [16]. As one kind of chloromethane, dichloromethane (DCM) has a saturated vapor pressure of $47.4 \mathrm{kPa}$ at $22^{\circ} \mathrm{C}$ and is a bit more volatile than acetone. We are curious whether the DCM adlayer between $\mathrm{MoS}_{2}$ and $\mathrm{SiO}_{2}$ substrate influences the Raman spectrum of $\mathrm{MoS}_{2}$. As shown in Fig. 7a-d, the height of $\mathrm{MoS}_{2}$ nanosheet was increased from 4.4 to 4.6, 4.8 and $5.2 \mathrm{~nm}$ after being incubated in DCM environment for 2, 3 and $6 \mathrm{~h}$, respectively. KPFM measurement indicated that the CPD of $\mathrm{MoS}_{2}$ was monotonically decreased from -73 to $-113 \mathrm{mV}$ with increased incubation time (Fig. 7e-h), implying the p-type doping effect of DCM on $\mathrm{MoS}_{2}$ [41]. In addition, the PL spectrum of $1 \mathrm{~L}$
$\mathrm{MoS}_{2}$ was increased after being incubated in DCM environment for 1 and $2 \mathrm{~h}$ (Fig. S19b), respectively, further confirming the p-type doping behavior of DCM. Similar to acetone and ethanol, DCM adlayer also showed obvious thickness-related influence on ULF breathing mode of $6 \mathrm{~L} \mathrm{MoS}_{2}$ nanosheet. As shown in Fig. 7i, j, the ULF breathing mode of $6 \mathrm{~L} \mathrm{MoS}_{2}$ nanosheet showed red shift, coexistence of red and blue shift, and blue shift with increased thickness of DCM adlayer, respectively, which was similar to that of $\mathrm{MoS}_{2}$ nanosheet with acetone adlayer. However, the high frequency Raman spectra of $6 \mathrm{~L}$ $\mathrm{MoS}_{2}$ nanosheet showed no obvious change (Fig. 7k). Aforementioned results demonstrated that the ULF breathing mode of $\mathrm{MoS}_{2}$ nanosheet was influenced by the thickness of organic adlayer rather than the doping effect.

Based on aforementioned results, we proposed a plot to illustrate the thickness influence of organic adlayer on the ULF breathing mode of $\mathrm{MoS}_{2}$ nanosheets. As shown in Fig. 8, ULF breathing mode in $\mathrm{MoS}_{2}$ nanosheets showed red shift when acetone and DCM adlayers were lower than 0.7 and $0.4 \mathrm{~nm}$, respectively. While blue shift of ULF breathing mode in $\mathrm{MoS}_{2}$ nanosheets was observed when acetone and DCM was thicker than 1.6 and $0.8 \mathrm{~nm}$, respectively. The coexistence of blue shift and red shift was observed when the thickness of acetone adlayer is in the range of 0.7 to $1.6 \mathrm{~nm}$. Such phenomenon was also found for DCM adlayer in the range of 0.4 to $0.8 \mathrm{~nm}$. The molecular heights of acetone and DCM are 0.26 and $0.24 \mathrm{~nm}$ (Fig. S21), respectively. While acetone has a length of $0.43 \mathrm{~nm}$, which is much larger than that of DCM. Such different critical thickness of acetone and DCM adlayer might be attributed to their different molecular size.

The red and blue shift of ULF breathing mode peak could be explained as follows. The red shift of ULF breathing mode in $\mathrm{MoS}_{2}$ nanosheet was also observed in heterostructures of $\mathrm{MoS}_{2}$ and graphene [47], which was attributed to the interfacial interaction between $\mathrm{MoS}_{2}$ and graphene. As previously reported, organic molecules such as THF and cyclohexane can form crystal-like adlayers in the confined space between graphene and mica substrate [24]. Theoretical simulation indicated that THF adlayer still exhibited solid-like behavior as the thickness increased [28]. Our previous report showed that even monolayer graphene can induce red shift of ULF breathing mode in $\mathrm{MoS}_{2}$ nanosheet [47]. After being incubated in acetone environment for a certain time, the intercalated acetone adlayer can be considered as substrate for $\mathrm{MoS}_{2}$ instead of the beneath $\mathrm{SiO}_{2}$ substrate. In this case, there could be interfacial interaction between 


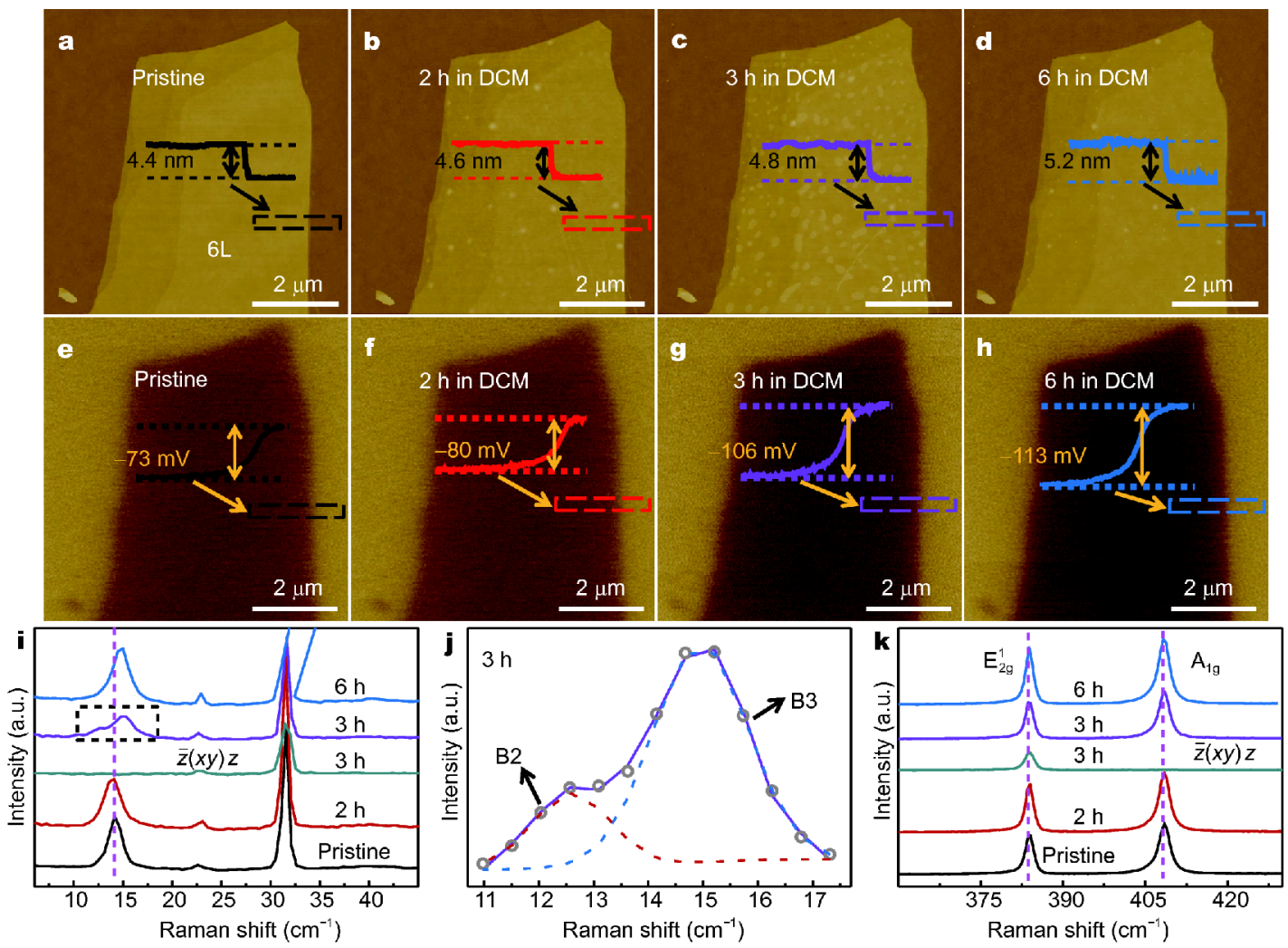

Figure 7 (a-d) AFM images of $6 \mathrm{~L} \mathrm{MoS}_{2}$ nanosheet (a) before and after being incubated in dichloromethane (DCM) environment for (b) 2 , (c) 3 and (d) $6 \mathrm{~h}$, respectively. (e-h) Corresponding KPFM images of $6 \mathrm{~L} \mathrm{MoS}_{2}$ nanosheet (e) before and after being incubated in acetone environment for (f) 2, (g) 3 and (h) 6 h, respectively. (i) ULF Raman spectra of $6 \mathrm{~L} \mathrm{MoS}_{2}$ before and after being incubated in DCM environment. (j) Magnified image of ULF breathing mode of $\mathrm{MoS}_{2}$ nanosheet shown in (i). (k) High frequency Raman spectra of $6 \mathrm{~L} \mathrm{MoS}$ before and after being incubated in DCM environment. Coexistence of red and blue shift of ULF breathing mode of $6 \mathrm{~L} \mathrm{MoS}_{2}$ nanosheet can be clearly observed after deconvolution.

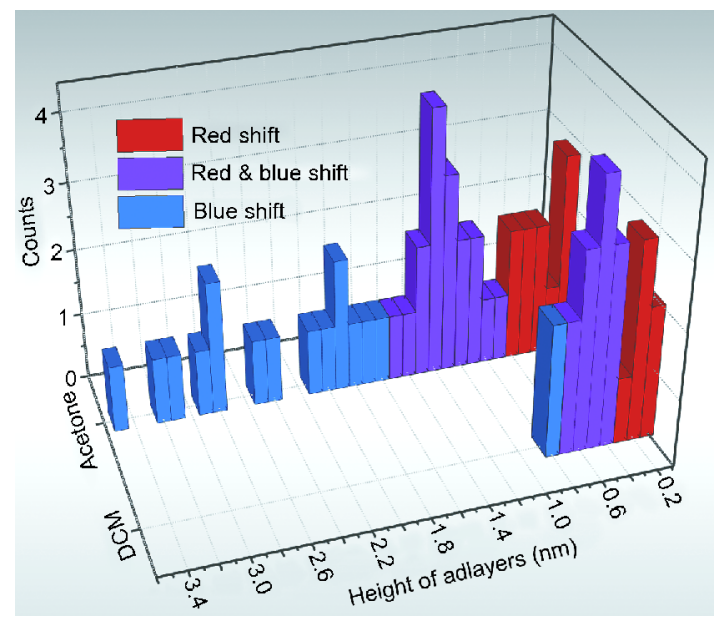

Figure 8 Histogram of the correlation between acetone and DCM adlayers thickness and the ULF breathing mode evolution of $\mathrm{MoS}_{2}$ nanosheets.

acetone adlayer and $\mathrm{MoS}_{2}$ nanosheet, which led to the red or blue shift of ULF breathing mode. When the thickness of acetone adlayer was $0.5 \mathrm{~nm}$, it could be comparable to that of monolayer graphene and might induce red shift of ULF breathing mode. When $\mathrm{MoS}_{2}$ nanosheet was put on few-layer graphene $(2 \mathrm{~L}-5 \mathrm{~L})$, coexistence of red and blue shift of ULF breathing mode was also observed [47]. Meanwhile, coexistence of red and blue shift of ULF breathing mode was also observed in our results, implying the organic adlayers play similar role in affecting the ULF breathing modes of $\mathrm{MoS}_{2}$ nanosheets to those of graphene layers. Therefore, as the acetone adlayer thickness exceeds $0.7 \mathrm{~nm}$ or more, we can image that the $6 \mathrm{~L}$ $\mathrm{MoS}_{2}$ nanosheet might be completely floated on acetone adlayer.

Besides $\mathrm{MoS}_{2}, \mathrm{WSe}_{2}$ nanosheets were also incubated in acetone environment to investigate the influence of acetone adlayer with various thicknesses on the ULF breathing mode. Fig. 9 shows the ULF Raman spectra of $4 \mathrm{~L} \mathrm{WSe}{ }_{2}$ nanosheet after being incubated in acetone environment for $1,2.5$ and $3 \mathrm{~h}$, respectively. With increased incubation time, the ULF breathing mode of $4 \mathrm{~L} \mathrm{WSe}_{2}$ 


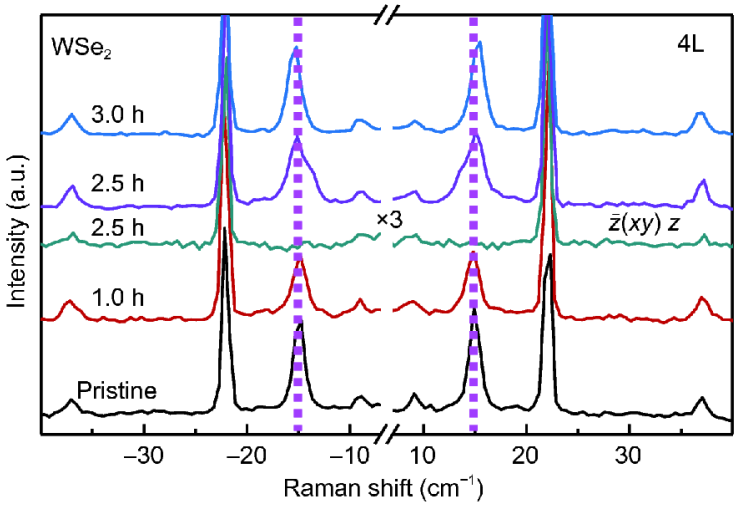

Figure 9 ULF Raman spectra of $4 \mathrm{~L} \mathrm{WSe} e_{2}$ nanosheet before and after being incubated in acetone environment for $1,2.5$ and $3 \mathrm{~h}$, respectively.

nanosheet also showed red shift, coexistence of red and blue shift, and blue shift, respectively, which is similar to those of $\mathrm{MoS}_{2}$. Such phenomena were also observed in $6 \mathrm{~L}$ $\mathrm{WSe}_{2}$ nanosheet incubated in acetone environment for different time (Fig. S22), implying the versatile influence of organic adlayer on the ULF breathing mode of TMDCs nanosheets.

\section{CONCLUSIONS}

In summary, the influence of organic adlayer thickness on the ULF breathing mode of TMDCs nanosheets was systematically investigated by AFM, KPFM as well as ULF Raman spectroscopy. With increased incubation time, AFM measurement showed increased thickness of organic adlayer. Raman spectroscopy indicated that the thickness of organic adlayer played an important role in affecting the ULF breathing mode of TMDCs nanosheets. When the thickness of acetone adlayer was lower than $0.7 \mathrm{~nm}$, only red-shifted ULF breathing mode was observed. As the thickness exceeded $1.6 \mathrm{~nm}$, blue-shifted ULF breathing mode was observed. While blue and red shifts coexisted as the thickness was measured in the range of 0.7 to $1.6 \mathrm{~nm}$. DCM adlayer also showed similar influence on the ULF breathing mode but lower thickness compared with that of acetone. KPFM measurement confirmed the enhanced $\mathrm{n}$-doping and $\mathrm{p}$-doping behaviors of acetone and DCM with increased thickness, respectively. Besides $\mathrm{MoS}_{2}$, ULF breathing mode of $\mathrm{WSe}_{2}$ nanosheet was also be influenced by the acetone adlayer, indicating the versatile influence of organic adlayer on the properties of TMDCs nanosheets. Our results not only show the influence of organic adlayer on the optical properties of TMDCs nanosheets, but also shine a pave to modulate the optoelectronic properties of TMDCs na- nosheets.

Received 22 April 2018; accepted 28 May 2018;

published online 28 June 2018

1 Balog R, Jørgensen B, Nilsson L, et al. Bandgap opening in graphene induced by patterned hydrogen adsorption. Nat Mater, 2010, 9: 315-319

2 Kozlov SM, Viñes F, Görling A. Bandgap engineering of graphene by physisorbed adsorbates. Adv Mater, 2011, 23: 2638-2643

3 Cao P, Varghese JO, Xu K, et al. Visualizing local doping effects of individual water clusters on gold(111)-supported graphene. Nano Lett, 2012, 12: 1459-1463

4 Fang H, Chuang S, Chang TC, et al. High-performance single layered $\mathrm{WSe}_{2}$ p-FETs with chemically doped contacts. Nano Lett, 2012, 12: 3788-3792

5 Fang H, Tosun M, Seol G, et al. Degenerate n-doping of few-layer transition metal dichalcogenides by potassium. Nano Lett, 2013, 13: 1991-1995

6 Goncher SJ, Zhao L, Pasupathy AN, et al. Substrate level control of the local doping in graphene. Nano Lett, 2013, 13: 1386-1392

7 Mouri S, Miyauchi Y, Matsuda K. Tunable photoluminescence of monolayer $\mathrm{MoS}_{2}$ via chemical doping. Nano Lett, 2013, 13: 59445948

8 Tongay S, Zhou J, Ataca C, et al. Broad-range modulation of light emission in two-dimensional semiconductors by molecular physisorption gating. Nano Lett, 2013, 13: 2831-2836

9 Dhakal KP, Duong DL, Lee J, et al. Confocal absorption spectral imaging of $\mathrm{MoS}_{2}$ : optical transitions depending on the atomic thickness of intrinsic and chemically doped $\mathrm{MoS}_{2}$. Nanoscale, 2014, 6: 13028-13035

10 Kiriya D, Tosun M, Zhao P, et al. Air-stable surface charge transfer doping of $\mathrm{MoS}_{2}$ by benzyl viologen. J Am Chem Soc, 2014, 136: 7853-7856

11 Yang L, Majumdar K, Liu H, et al. Chloride molecular doping technique on 2D materials: $\mathrm{WS}_{2}$ and $\mathrm{MoS}_{2}$. Nano Lett, 2014, 14: 6275-6280

12 Zhao P, Kiriya D, Azcatl A, et al. Air stable p-doping of $\mathrm{WSe}_{2}$ by covalent functionalization. ACS Nano, 2014, 8: 10808-10814

13 Andleeb S, Kumar Singh A, Eom J. Chemical doping of $\mathrm{MoS}_{2}$ multilayer by p-toluene sulfonic acid. Sci Tech Adv Mater, 2015, 16: 035009

14 Jing Y, Tang Q, He P, et al. Small molecules make big differences: molecular doping effects on electronic and optical properties of phosphorene. Nanotechnology, 2015, 26: 095201

15 Cai Y, Zhou H, Zhang G, et al. Modulating carrier density and transport properties of $\mathrm{MoS}_{2}$ by organic molecular doping and defect engineering. Chem Mater, 2016, 28: 8611-8621

16 Choi J, Zhang H, Du H, et al. Understanding solvent effects on the properties of two-dimensional transition metal dichalcogenides. ACS Appl Mater Interfaces, 2016, 8: 8864-8869

17 Singh D, Gupta SK, Sonvane Y, et al. Modulating the electronic and optical properties of monolayer arsenene phases by organic molecular doping. Nanotechnology, 2017, 28: 495202

18 Li L, Pi LJ, Li HQ, et al. Photodetectors based on two-dimensional semiconductors: Progress, opportunity and challenge. Chin Sci Bull, 2017, 62: 3134-3153

19 Huang Y, Zhuge F, Hou J, et al. Van der Waals coupled organic molecules with monolayer $\mathrm{MoS}_{2}$ for fast response photodetectors with gate-tunable responsivity. ACS Nano, 2018, 12: 4062-4073 
20 Kufer D, Konstantatos G. Highly sensitive, encapsulated $\mathrm{MoS}_{2}$ photodetector with gate controllable gain and speed. Nano Lett, 2015, 15: 7307-7313

21 Zhang W, Huang JK, Chen $\mathrm{CH}$, et al. High-gain phototransistors based on a CVD $\mathrm{MoS}_{2}$ monolayer. Adv Mater, 2013, 25: 3456-3461

22 Lin JD, Han C, Wang F, et al. Electron-doping-enhanced trion formation in monolayer molybdenum disulfide functionalized with cesium carbonate. ACS Nano, 2014, 8: 5323-5329

$23 \mathrm{Xu} \mathrm{K}$, Cao P, Heath JR. Graphene visualizes the first water adlayers on mica at ambient conditions. Science, 2010, 329: 1188-1191

24 Cao P, Xu K, Varghese JO, et al. Atomic force microscopy characterization of room-temperature adlayers of small organic molecules through graphene templating. J Am Chem Soc, 2011, 133: 2334-2337

25 Komurasaki H, Tsukamoto T, Yamazaki K, et al. Layered structures of interfacial water and their effects on Raman spectra in graphene-on-sapphire systems. J Phys Chem C, 2012, 116: 1008410089

26 Lee MJ, Choi JS, Kim JS, et al. Characteristics and effects of diffused water between graphene and $\mathrm{a} \mathrm{SiO}_{2}$ substrate. Nano Res, 2012, 5: 710-717

27 Shim J, Lui CH, Ko TY, et al. Water-gated charge doping of graphene induced by mica substrates. Nano Lett, 2012, 12: 648-654

28 Chen S, Li H, Cao P, et al. Understanding liquid-solid-like behavior of tetrahydrofuran adlayers at room temperature between graphene and mica: a Born-Oppenheimer molecular dynamics study. J Phys Chem C, 2013, 117: 21894-21900

29 Varghese JO, Agbo P, Sutherland AM, et al. The influence of water on the optical properties of single-layer molybdenum disulfide. Adv Mater, 2015, 27: 2734-2740

30 Lee DE, Ahn G, Ryu S. Two-dimensional water diffusion at a graphene-silica interface. J Am Chem Soc, 2014, 136: 6634-6642

31 Zhang Q, Peng B, Chan PKL, et al. A pentacene monolayer trapped between graphene and a substrate. Nanoscale, 2015, 7: 1466314668

32 Bampoulis P, Witteveen JP, Kooij ES, et al. Structure and dynamics of confined alcohol-water mixtures. ACS Nano, 2016, 10: 67626768

33 Voïtchovsky K, Giofrè D, José Segura J, et al. Thermally-nucleated self-assembly of water and alcohol into stable structures at hydrophobic interfaces. Nat Commun, 2016, 7: 13064

34 Mak KF, He K, Lee C, et al. Tightly bound trions in monolayer $\mathrm{MoS}_{2}$. Nat Mater, 2012, 12: 207-211

35 Guo W, Cheng C, Wu Y, et al. Bio-inspired two-dimensional nanofluidic generators based on a layered graphene hydrogel membrane. Adv Mater, 2013, 25: 6064-6068

36 Guo W, Jiang L. Two-dimensional ion channel based soft-matter piezoelectricity. Sci China Mater, 2014, 57: 2-6

37 Gao J, Feng Y, Guo W, et al. Nanofluidics in two-dimensional layered materials: inspirations from nature. Chem Soc Rev, 2017, 46: 5400-5424
38 Ji J, Kang Q, Zhou Y, et al. Osmotic power generation with positively and negatively charged 2D nanofluidic membrane pairs. Adv Funct Mater, 2016, 27: 1603623

39 Cheng H, Zhou Y, Feng Y, et al. Electrokinetic energy conversion in self-assembled 2D nanofluidic channels with janus nanobuilding blocks. Adv Mater, 2017, 29: 1700177

40 Wang L, Feng Y, Zhou Y, et al. Photo-switchable two-dimensional nanofluidic ionic diodes. Chem Sci, 2017, 8: 4381-4386

41 Feng Y, Zhang K, Li H, et al. In situ visualization and detection of surface potential variation of mono and multilayer $\mathrm{MoS}_{2}$ under different humidities using Kelvin probe force microscopy. Nanotechnology, 2017, 28: 295705

42 Li Y, Xu CY, Hu PA, et al. Carrier control of $\mathrm{MoS}_{2}$ nanoflakes by functional self-assembled monolayers. ACS Nano, 2013, 7: 77957804

43 Rice C, Young RJ, Zan R, et al. Raman-scattering measurements and first-principles calculations of strain-induced phonon shifts in monolayer $\mathrm{MoS}_{2}$. Phys Rev B, 2013, 87: 081307

44 Lin H, Schilo A, Kamoka AR, et al. Insight into the wetting of a graphene-mica slit pore with a monolayer of water. Phys Rev B, 2017, 95: 195414

45 Buscema M, Steele GA, van der Zant HSJ, et al. The effect of the substrate on the Raman and photoluminescence emission of single-layer $\mathrm{MoS}_{2}$. Nano Res, 2014, 7: 561-571

46 Yu Y, Yu Y, Xu C, et al. Engineering substrate interactions for high luminescence efficiency of transition-metal dichalcogenide monolayers. Adv Funct Mater, 2016, 26: 4733-4739

$47 \mathrm{Li} \mathrm{H}, \mathrm{Wu} \mathrm{JB}$, Ran $\mathrm{F}$, et al. Interfacial interactions in van der Waals heterostructures of $\mathrm{MoS}_{2}$ and graphene. ACS Nano, 2017, 11: $11714-11723$

Acknowledgements This work was supported by the National Natural Science Foundation of China (21571101 and 51322202), the Natural Science Foundation of Jiangsu Province in China (BK20161543 and BK20130927), the Joint Research Fund for Overseas Chinese, Hong Kong and Macao Scholars (51528201), and the Natural Science Foundation of Jiangsu Higher Education Institutions of China (15KJB430016).

Author contributions $\mathrm{Li} \mathrm{H}$ designed the project. Li H, Huang X and Huang $\mathrm{W}$ guided the project. Wu S performed the experiments. Shi X, Liu Y, Wang L, Zhang J, Zhao W and Wei P performed some supplemental experiments. $\mathrm{Li} \mathrm{H}$ and $\mathrm{Wu} \mathrm{S}$ analyzed results and wrote the manuscript. All authors participated in general discussion of the paper.

Conflict of interest The authors declare that they have no conflict of interest.

Supplementary information Supporting data are available in the online version of the paper. 

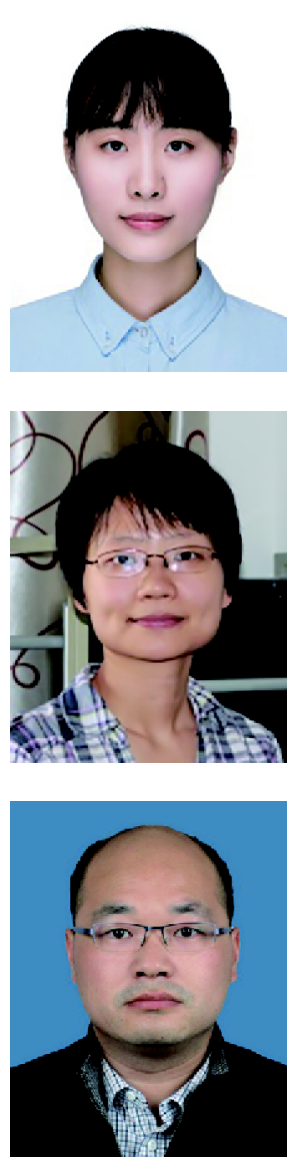

Shiyu Wu is a master student at the Institute of Advanced Materials (IAM), Nanjing Tech University. Her research interest focuses on the synthesis and characterization of two-dimensional materials.
Xiao Huang is currently a professor at the IAM, Nanjing Tech University. She received her bachelor's degree from the School of Materials Science and Engineering at Nanyang Technological University in Singapore in 2006 and completed her PhD in 2011 under the supervision of Prof. Hua Zhang and Prof. Freddy Boey. Her research interest includes the synthesis and applications of two-dimensional nanomaterial-based hybrids.
Hai Li is currently a professor at the IAM, Nanjing Tech University. He received his bachelor degree from Zhengzhou University in 2001 and completed his PhD in 2007 under the supervision of Prof. Jun Hu. He worked as postdoctoral fellow in Prof. Hua Zhang's group in the School of Materials Science and Engineering at Nanyang Technological University in Singapore from 2009 to 2014. His research interest includes the synthesis, characterization and applications of two-dimensional materials and their heterostructures.

\section{二维有机分子吸附层对过渡金属硫化物纳米薄片超低波拉曼光谱的影响}

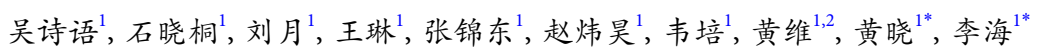

摘要 在二维材料下表面与衬底之间的受限空间中, 物理吸附物如水分子和有机分子等可形成二维吸附层. 然而, 这类吸附层如何影响其 上层二维材料的性能尚未被探究. 本文中, 我们结合原子力显微镜、开尔文力显微镜以及超低波拉曼光谱仪来探究有机分子吸附层对其 上的少层二硫化钿及二硒化铇纳米薄片性质的影响. 随吸附层厚度增加, 纳米薄片的超低波呼吸模式拉曼峰发生红移、红移和蓝移共存 以及仅有蓝移的现象. 此外, 纳米薄片的掺杂程度也逐渐增强. 理解有机分子吸附层与二维材料下表面之间的相互作用, 有望对二维材料 性质的调节提供帮助. 